\title{
Attentional and perceptual asymmetries in an immersive decision-making task
}

\author{
Stefanie Klatt ${ }^{1} \cdot$ Paul R. Ford ${ }^{2} \cdot$ Nicholas J. Smeeton $^{2}$ \\ Published online: 5 December 2019 \\ (C) The Psychonomic Society, Inc. 2019
}

\begin{abstract}
Pseudoneglect represents the tendency in healthy people to show a slight bias in favour of stimuli appearing in the left visual field. Some studies have shown that this leftward bias can be annulled or reserved towards a rightward bisection bias when lateral attentional biases are assessed in far space. Using an immersive simulated, ecologically valid football task, we investigated whether possible attentional and perceptual asymmetries affect sport-specific decision making. Twenty-seven sport athletes were required to judge different game situations, which involved both perceptual and attentional skills to perceive player configurations in the visual periphery. We did not find any performance differences in accuracy rate between the left and right visual field side for stimuli presented close to the screen centre in an object-detection (perception-based) and feature-recognition (attentionbased) task. This result is in line with previous findings showing an absence of a left- or rightward bisection bias in far space. However, accuracy was higher for stimuli being presented at visual angles wide away from the screen centre at the left side compared to the right side of visual field. This finding cannot be explained by literature focusing on pseudoneglect in far space, but rather by previous findings on landmark judgments often showing left bias both in near and in far space. Overall, the current findings provide new perspectives on attentional and perceptual asymmetries in real-world scenarios, and different interpretations of results are discussed.
\end{abstract}

Keywords Ipsilesional side $\cdot$ Leftward bias $\cdot$ Line bisection $\cdot$ Pseudoneglect

\section{Introduction}

Over the last decades, much research has dealt with visual neglect following parietal lobe damage to the brain. Researchers have demonstrated that affected individuals usually bisect horizontal lines away from the centre point and on the ipsilesional lobe side (i.e. the intact side). This effect is thought to result from ignorance of the contralesional side of the stimulus or their hyper-attentiveness to the ipsilesional side (Driver \& Mattingley, 1998; Jewell, \& McCourt, 2000; Varnava, \& Halligan, 2008). However, the majority of healthy people mis-bisect lines as well, a phenomenon referred to as 'pseudoneglect' (Bowers \& Heilman, 1980). Commonly manual line bisection or task variants (e.g. landmark task) are used

Stefanie Klatt

s.klatt@dshs-koeln.de

1 Institute of Exercise Training and Sport Informatics, German Sport University Cologne, Cologne, Germany

2 University of Brighton, Brighton, UK to diagnose visuospatial neglect/pseudoneglect (cf. Fink, Marshall, Weiss, Toni, \& Zilles, 2002; McCourt \& Jewell, 1999). The majority of previous studies have intended to bisect spatial areas (e.g. midpoint between two goalposts, cf. Nicholls, Loetscher, \& Rademacher, 2010). While some research has been conducted on visual-field advantages for identifying targets in rapid serial visual presentation (for a review, see Verleger \& Śmigasiewicz, 2015), the effect of such perceptual asymmetries for stimuli presented at different visual angles in the visual periphery on people's decision making has not yet been investigated.

Pseudoneglect was first documented by Bowers and Heilman (1980) and represents a visuospatial bias in favour of the left side of space. Healthy people usually respond quicker and judge stimuli as larger and brighter that appear in the left visual field compared to the right visual field (Jewell $\&$ McCourt, 2000). Although pseudoneglect seems to arise from anatomical and functional asymmetries of the brain networks subserving visuospatial attention (Bultitude \& Aimola Davies, 2006; Corbetta \& Shulman, 2011), the phenomenon remains to be fully mapped onto brain activity. The lateralization of cognitive functions in the human brain facilitates 
optimal information processing (Cai, van der Haegen, \& Brysbaert, 2013; Corballis, 1989).

For some time now, there has been general agreement in the scientific literature that brain lateralization is not a unique characteristic of humans, but that it is present in a wide range of vertebrate species (cf. Rogers, Vallortigara, \& Andrew, 2013) as well as in some invertebrates (e.g. Frasnelli et al., 2012). From an evolutionary perspective, there is research involving several classes of vertebrate animals showing that the brain's different hemispheres evolved to predominantly serve different functions (e.g. Rogers, Zucca, \& Vallortigara, 2004). Whereas the left hemisphere - hence right visual field mainly controls routine and goal-directed action behaviour as, for example, singing in birds (Nottebohm, Stokes, \& Leonard, 1976) or catching prey in toads (Vallortigara, Rogers, Bisazza, Lippolis, \& Robins, 1998), the right hemisphere -hence left visual field - is more activated when something unexpected happens, as for example, the appearance of a predator (MacNeilage, Rogers, \& Vallortigara, 2009). For instance, it was found that toads only reacted to a snake rubber head by jumping away if the head was pushed towards them from the left but not when approaching from the right. Hence, the likelihood of the perception of a certain relevant stimulus is influenced by its location (Lippolis, Bisazza, Rogers, \& Vallortigara, 2002). Further research has dealt with preferential use of the left or right visual hemifield in vertebrates when searching for food or escape from predators (e.g. Vallortigara \& Rogers, 2005).

The consideration of the two hemispheres as separate cognitive units traces back to research that demonstrated differences in the representation of cognitive processes when examining the hemispheres separately. The different processing strategies of the two hemispheres (left, right) can, for example, be demonstrated using visual discrimination tasks in which the dominance of the right hemisphere for analysing visuospatial patterns can be examined by separately presenting contents in the left and right visual field (cf. Milner, 1975). Holtzman, Sidtis, Volpe, Wilson, and Gazzaniga (1981), among others, found that either hemisphere can direct attention to a point in either the left or right visual field. A right hemispheric dominance in spatial attention leads to an expansion of the contralateral left visual field (cf. Fink et al., 2000) and therefore explains the fact that healthy people normally perceive the left half of a line as longer than the right half (cf. Bultitude \& Aimola Davies, 2006).

However, the phenomenon described above only applies to line-bisection tasks in the near space. There is evidence that the usual leftward bias becomes a rightward bias when lateral attentional biases are assessed in far space (beyond arm reach; cf. Longo \& Lourenco, 2006, 2007). Researchers have observed that healthy people make greater leftward errors when they bisect lines in near versus far space (e.g. Dellatolas, Vanluchene, \& Coutin, 1996; Varnava, McCarthy, \&
Beaumont, 2002). Justifications of the mis-bisection changing by distance (lines in near space are bisected to the left, lines in far space are bisected to the right; Longo \& Lourenco, 2006) are usually explained by a mechanism that processes near and far space differently via different cortical areas (e.g. Weiss et al., 2000).

There are some studies that have analysed the effects of pseudoneglect in near and far space in real-life situations/ tasks showing that lateral attentional biases appear on other experimental tests of line bisection. The implication of these results is that the pseudoneglect phenomenon has consequences for everyday behaviours, such as driving (e.g. Benedetto, Pedrotti, Bremond, \& Baccino, 2013) and sporting performance (e.g. Masters, van der Kamp, \& Jackson, 2007; Nicholls et al., 2010). There is research, for example, that has shown lateralized bias with regard to collisions, whereby people bump into doors on the right-hand side more often than on the left (e.g. Nicholls, Loftus, Orr, \& Barre, 2008). There are few studies that have been conducted in the area of sports investigating the effect of attentional asymmetries (for a review, see Churches \& Nicholls, 2016). To date, these studies have shown that far space has a rightward bias in performance outcome. Roberts and Turnbull (2010) investigated whether lateral biases in visual attention affect putting performance in golf. They found more misses to the right side of the hole when putting a golf ball from $2.26 \mathrm{~m}$. Nicholls et al. (2010) observed asymmetries in Australian Rules football and also confirmed the findings of Longo and Lourenco (2006) of a rightward bias in far space: When kicking the ball towards the right side of the goal players missed more often than when kicking the ball to the left. Football studies of Masters et al. (2007) as well as of Weigelt and Memmert (2012) confirmed previous findings of a rightward bias for goal kicking when showing that players kicked the ball into the right side of the net more often when the goalkeeper was placed in the middle of the goal.

So far, studies dealing with attentional asymmetries in the area of sport have mainly focused on tasks that are closely linked to line-bisection tasks, for example, in penalty shootouts where participants should kick the ball exactly in between two goal posts in far space (cf. Nicholls et al., 2010; Weigelt \& Memmert, 2012). The task being used in the current study differs from the earlier ones in that did not intend to find the midpoint of a line or of any other more naturalistic situations, such as the midpoint of a goal, but rather intended to analyse perceptual and attentional capabilities to extract information in the left and right visual field in a representative sport-specific task. To what extent attentional and perceptual capabilities in the periphery and, thus, also decision making, are influenced by attentional asymmetries has, to the best of our knowledge, not yet been the focus of any research investigation.

Furthermore, this study aimed to address the lack of research addressing the differences in perceptual and attentional 
asymmetries. Although the concepts of visual perception and visual attention are closely related and some studies do not really define these terms clearly, there are substantial differences between the two concepts. In general, visual attention is a prerequisite for conscious recognition of information (cf. Dehaene, Changeaux, Naccache, Sackur, \& Sergent, 2006) and the visual focus of attention is allocated across parts of the visual field (Hüttermann \& Memmert, 2017). According to one line of thinking (e.g. Lamme, 2003; van Boxtel, Tsuchiya, \& Koch 2010, for differing views), people can only consciously perceive those objects/events onto which they direct their attention at a given time. A number of studies have demonstrated that the attentional focus is smaller than the visual field, i.e. perceptual capabilities are spread along a wider peripheral area than attentional capabilities (for a review, see Hüttermann \& Memmert, 2017). Especially in fast-paced team sports played on wide pitches and courts, such as football, a wide focus of attention and high levels of perceptual-cognitive skills are necessary in order to perform successfully and to make the best decision (Hüttermann, Helsen, Put, \& Memmert, 2018; Hüttermann, Memmert, \& Nerb, 2019; Hüttermann, Noël, \& Memmert, 2017).

Only recently, research has been published examining the link between decision making, visual attention, and perception in a sport-specific task (cf. Hüttermann, Ford, Williams, Vagas, \& Smeeton, 2019a; Hüttermann, Smeeton, Ford, \& Williams, 2019b). The authors developed a football-specific decisionmaking task and investigated the attentional and perceptual capabilities of sport athletes. The positioning of team-mates and opponent players was varied using different distances between the players' formations, and participants were required to identify specific characteristics (e.g. running direction, jersey colour) of players. Results showed an attentional performance decrease with increasing viewing angles and confirmed previous research showing that attentional capabilities are less available in visual periphery than perceptual capabilities (cf. Hüttermann, Memmert, \& Simons, 2014). However, in these studies, peripheral stimuli presented on the left and right sides of participants' visual fields were evaluated together, i.e. responses were only rated as correct if stimuli on the left and right side were both correctly identified. This procedure prevented checking for any asymmetrical differences in perception and attention and the effect of the athletes' decision making.

Using the same method as Hüttermann and colleagues (cf. Hüttermann, Ford, et al., 2019a; Hüttermann, Smeeton, et al., 2019b), the current study aimed to examine asymmetrical differences in decision making, as well as attentional and perceptual capabilities. Participants performed a football-specific task that included in each trial feature-recognition, object-detection, and decision making. In order to create as realistic game situations as possible, the study was performed in a large immersive dome screen (creating an immersive $210^{\circ}$ stimulus projection environment around the participant). While the object-detection task required the differentiation between jersey colours (recognition of number of players wearing white jerseys), the feature-recognition task required the differentiation between colour and shape of stimuli (recognition of players wearing black jerseys and assessment of their running direction), thereby demanding visual attention (cf. Hüttermann, Ford, et al., 2019a; Hüttermann, Smeeton, et al., 2019b). The decision-making task required the choice of passing the ball to an "open" team-mate on either the left or right side or not. Based on the pseudoneglect phenomenon being observed in far space (cf. Longo \& Lourenco, 2006; Lourenco \& Longo, 2009), we expected higher accuracy rates in the feature-recognition and object-detection task for stimuli presented on the right side of the participants' visual field compared to their left side. Furthermore, we expected to find more correct decisions when stimuli were presented close compared to more distant from the participants' central fixation point (i.e. middle of the immersive screen).

\section{Method}

\section{Participants}

Altogether, 27 participants (seven female) aged 19-27 years ( $M_{\text {age }}=23.22$ years, $S D=2.03$ years) took part in the experiment, which was conducted in England. Data from three additional participants were excluded due to accuracy rates under chance level in all three subtasks. At the time of data collection, participants were regularly active in a team sport and regularly participated in competitions. Primary sports included football $(n=11)$, lacrosse ${ }^{1}(n=9)$, and basketball $(n=7)$. All participants reported normal or corrected-to-normal (with contact lenses) vision. Wearers of glasses had to be excluded from the study because their whole visual field is usually not covered by glasses. Twenty-four participants reported preferring to kick the ball with their right foot and three with their left foot. The study was carried out in accordance with the Helsinki Declaration of 1975, and written informed consent was obtained from each participant prior to testing. Approval was obtained from the lead institution's ethics board.

\section{Procedure}

Participants were tested individually in a laboratory room. They completed a manual line-bisection task and the football-specific task; the order in which these two tasks were administered was counterbalanced across participants. For the

\footnotetext{
${ }^{1}$ In the team sport lacrosse, two teams have to hit a small rubber ball into the opposing team's goal with the use of a crosse (a stick with a mesh at the end that is used to carry or catch the ball). It is a team's objective to get the ball into the crosse and to toss the ball into the goal while dodging the opposing players and their goalkeeper.
} 
manual line-bisection task, participants sat at a standard desk. For the completion of the football-specific task, participants stood $3 \mathrm{~m}$ in front of a $210^{\circ}$ curved projection screen (IGLOO Vision ltd, Shropshire, UK, radius of $3 \mathrm{~m}$, height: $2.20 \mathrm{~m}$; see Fig. 1).

Line bisection task The paper line-bisection task consisted of eight horizontal lines on a white paper sheet. Line length was on average $10.25 \mathrm{~cm}$ and ranged from 5 to $16 \mathrm{~cm}$ (cf. procedure of Noël, van der Kamp, Weigelt, \& Memmert, 2015). The lines were arranged in a way that none of the veridical centres of the lines were at the same horizontal position. Participants used a fine pencil to bisect lines.

Football-specific decision-making task We used an adapted version of the football-specific decision-making task developed by Hüttermann and colleagues (cf. Hüttermann, Ford, et al., 2019a; Hüttermann, Smeeton, et al., 2019b). The task was presented in Delphi XE 3. Instructions were delivered on the screen, but participants were also encouraged to ask questions prior to starting. Participants performed 24 trials preceded by two additional practice trials. After the presentation of a central fixation cross $(1,000 \mathrm{~ms})$, two stimuli were presented for $300 \mathrm{~ms}$ equidistant to and at opposite sides of the fixation cross in each trial (see Fig. 2). In order to cover a wide range of visual angles in the analysis, stimuli were presented at the edges of one of eight visual angles $\left(20^{\circ}, 40^{\circ}, 60^{\circ}, 80^{\circ}, 100^{\circ}\right.$, $120^{\circ}, 140^{\circ}$, and $160^{\circ}$ ) in a randomised order and equidistant to the left and right of the centre of the screen; see Hüttermann, Ford, et al., 2019a as well as Hüttermann, Smeeton, et al., $2019 \mathrm{~b}$, for a similar procedure). Each stimulus consisted of configurations of a maximum of four players at either side: One team-mate was presented on each side of the participant's visual field flanked by zero, one, two, or three opposing players chosen at random. These opposing players were always depicted moving towards the respective team-mate on

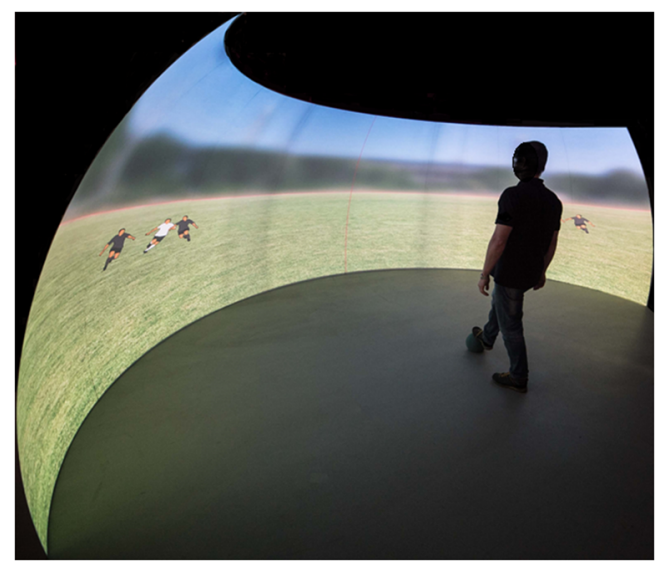

Fig. 1 The figure shows the experimental setup with a subject standing in front of the $2.4 \times 6.0 \mathrm{~m}$ IGLOO dome (the subject's face has been blurred for publication for privacy reasons)

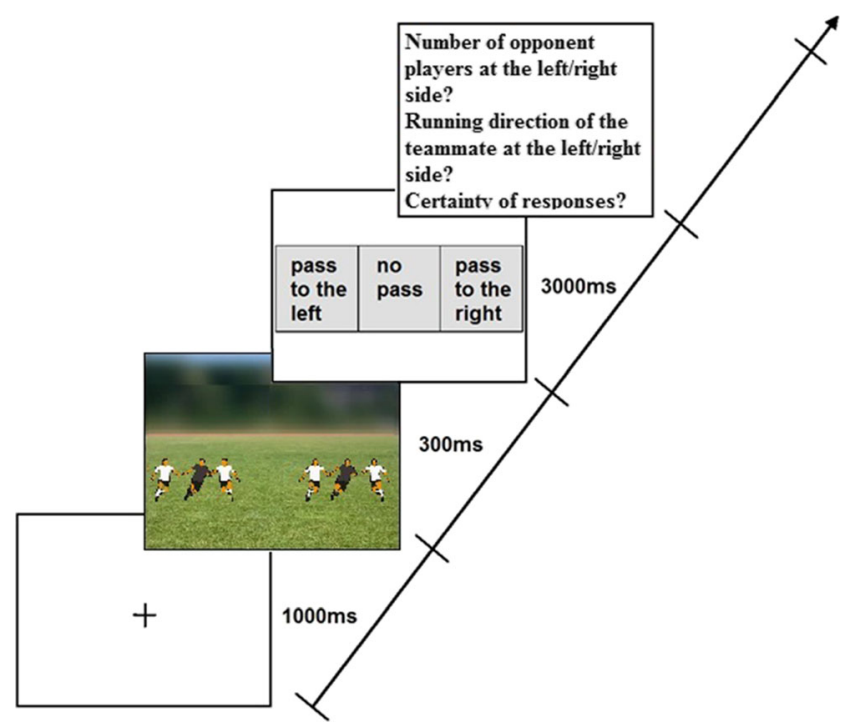

Fig. 2 Sequence of events in one exemplary trial (adapted from Hüttermann, Ford, et al., 2019a). The experimental trial shows a playing situation with the team-mate at the participant's left side running towards the centre and at his/her right side running towards the side line. As there are two opponent players at the left and two at the right side, the participant should decide not to pass the ball

each side of the participant's visual field, whereas the teammates moved either in the direction towards the side line (outer end of the screen) or towards the centre of the screen. All players were presented with a height of about $30 \mathrm{~cm}$.

Participants were told to imagine being the player in possession of the ball and were required to decide to play the ball either to an open team-mate to the left or right side of their visual field or to stop/control the ball (decision-making task). Only if they believed they had seen an open team-mate depicted running in their direction (towards the centre) and not being flanked by an opponent player, should they decide to pass the ball to this team-mate. Team-mates who were either running towards the side line or being flanked by at least one opponent player should not receive the ball. Participants had to make the decision by a sport-specific motor response (pass to the left, pass to the right, no pass) as quickly as possible, but at least within a timeframe of $3 \mathrm{~s}$. A ball was placed on the floor in front of the participants on each trial. They were required to pass the ball with their preferred foot in the direction of the freestanding team-mate on the left or right side or to put their foot on the ball if there was no free team-mate running towards the centre on both sides of the screen (cf. Fig. 1). Furthermore, directly following their decision, participants were required to verbally report the team-mates' running direction for each side (feature-recognition task) and verbally report the number of opponent players flanking their teammate on each side (object-detection task). They were prompted by messages on the screen (cf. Fig. 2) and gave their responses verbally to the experimenter who recorded their responses. The order of the requests for the left and right side 
occurred at random counterbalanced across all trials. Finally, following these verbalisations in the inter-trial period, participants were required to state how certain they were about their responses in the feature-recognition, object-detection, and decision-making tasks for each trial on a 10-point Likert scale ranging from 1 (very uncertain) to 10 (very certain).

\section{Data analysis}

Line-bisection task In the paper line-bisection task, the distance from the paper line's midpoint was measured in millimetres. The task was scored such that positive values indicate a bias to the right of the veridical centre, whereas negative values indicate a bias to the left. A one-sample t-test was used to compare the mean distance from the midpoint or zero (i.e. the actual midpoint was defined as zero; cf. procedure of Noël et al., 2015).

Spearman's Rho was used to calculate the relationships between scores in the line-bisection task and participants' scores in the football-specific task.

Football-specific task As in previous research using the football decision-making task (cf. Hüttermann, Ford, et al., 2019a; Hüttermann, Smeeton, et al., 2019b), we determined the participants' total score including only the trials in which participants performed successfully in all three tasks, i.e. making the right decision, correctly identifying the running direction of team-mates, and giving the correct number of opponent players for both sides of the visual field. We conducted a repeated-measures ANOVA with accuracy rate as the dependent variable and visual angle (narrow: $20^{\circ}, 40^{\circ}, 60^{\circ}$, and $80^{\circ}$; wide: $100^{\circ}, 120^{\circ}, 140^{\circ}$, and $\left.160^{\circ}\right)^{2}$ as the within-participant factor.

In addition, we evaluated the data of each single subtask (decision making, feature recognition, object detection). ${ }^{3}$ For the decision-making task, we again performed a repeatedmeasures ANOVA with accuracy rate as the dependent variable and visual angle as the within-participant factor. In contrast to the decision-making task, in which only one response (pass to the left, pass to the right, or no pass) was required, for the feature-recognition and object-detection subtasks, we conducted separate repeated-measures ANOVAs with accuracy rate as the dependent variable and visual angle (narrow, wide) and visual field side (left, right) as within-participant factors.

\footnotetext{
$\overline{2}$ Please note that the term "narrow visual angle" applies to angles in real-life situations in this context and not to angles in computer-based laboratory tasks where such angles would already be defined as wide ones.

${ }^{3}$ To avoid any misunderstandings it should be clarified that participants performed only one football-specific task, i.e. they were shown only one game situation in every trial. The categorization into a decision-making, featurerecognition, and object-detection task should only help to analyse the data in more detail by distinguishing between different cognitive skills, such as perceptual, attentional, and decision-making skills.
}

A Bonferroni-corrected method was performed for post hoc testing of significant main effects.

\section{Results}

Line bisection task A one-sample t-test comparing the mean distance from the midpoint on the paper line-bisection task to zero showed a significant bias to the left, $t(26)=-17.7, p<$ $.001, d=3.40$. The distance to the left side was $0.9 \%(S D=$ $0.3 \%$ ) of the total length of the line. We did not find any significant correlation between participants' scores in the line-bisection task and their accuracy in the football-specific task nor in one of the three subtasks (all ps > .05).

Total score football-specific task In the total score, accuracy rate was defined as a combination of correct responses in the feature recognition, object detection, and decision-making tasks. Participants correctly evaluated $47.2 \%(S D=9.3 \%)^{4}$ of the trials. Accuracy differed as a function of visual angle, $F(1,26)=81.3, p<.001, \eta^{2}=.76$; participants performed better when they had to judge game situations in the narrowangle condition $\left(20-80^{\circ} ; M=67.3 \%, S D=15.3 \%\right)$ compared to the wide-angle condition $\left(100-160^{\circ} ; M=27.2 \%, S D=\right.$ $14.4 \%$ ). Figure 3 demonstrates the accuracy along each visual angle.

Decision-making subtask In total, participants made the correct decision (pass to the left, no pass, pass to the right) on $91.2 \%(S D=7.6 \%)$ of all trials independent of their performances in the feature-recognition and object-detection task. Decision-making performance decreased with increasing visual angle of stimuli, resulting in a significant main effect for the factor visual angle (narrow: $M=98.2 \%, S D=3.5 \%$; wide: $M=84.3 \%, S D=15.2 \%), F(1,26)=20.3, p<.001, \eta^{2}=.44$ (see Fig. 4). Overall, participants reported certainty rates of 7.7 $(S D=0.8)$ with regard to their decisions on a 10-point Likert scale that decreased from narrow $(M=8.3, S D=0.8)$ to wide visual angles $(M=7.1, S D=1.1), F(1,26)=56.0, p<.001, \eta^{2}$ $=.68$.

Feature recognition subtask In the feature-recognition task (i.e. identification of the team-mates' running direction; attention-based task), the ANOVA revealed a significant main effect for visual angles, $F(1,26)=46.4, p<.001, \eta^{2}=.64$, indicating a decline of performance from narrow to wide

\footnotetext{
${ }^{4}$ Although this accuracy rate seems to be very low at first sight, it should be considered that a trial was only classified as correct if participants made the correct decision and gave correct answers in the object-detection and featurerecognition task - also including situations that required visual angles of up to $160^{\circ}$, i.e. lying outside the maximal shift of attention measured in previous research (e.g. Hüttermann et al., 2014; Hüttermann, Memmert, Simons, \& Bock, 2013).
} 


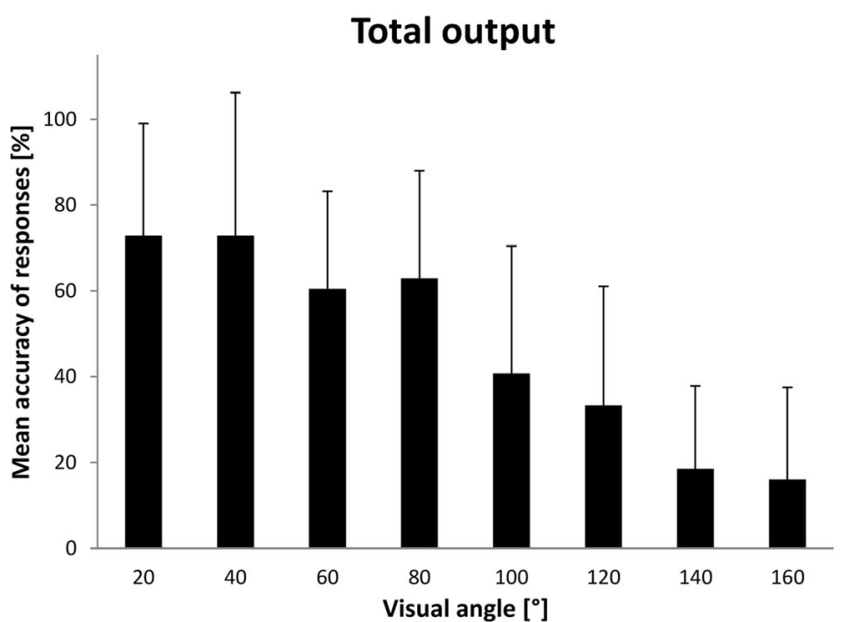

Fig. 3 Percentage of participants' total accuracy rate (including correct responses in the decision-making, feature-recognition, and objectdetection subtask) in degrees of visual angle. Symbols represent acrossparticipant means and error bars represent standard deviations

visual angle. In addition, participants showed higher accuracy rates along the left visual field side $(M=72.5 \%, S D=9.6 \%)$ compared to the right side $(M=68.1 \%, S D=8.3 \%), F(1,26)=$ $\left.4.4, p=.047, \eta^{2}=.14\right)$. Figure 5 presents the accuracy rate for each visual angle as a function of visual field side (see top graph of Fig. 5). We did not find an interaction effect for visual angle and visual field side, $F(1,26)=2.2, p=.147$.

In spite of the absent interaction, we performed additional $\mathrm{t}$-tests to check if the asymmetrical performance differences in visual attention existed for stimuli presented both near (mean accuracy rate for visual angles of $20^{\circ}, 40^{\circ}, 60^{\circ}$, and $80^{\circ}$ ) and far (wide) away (mean accuracy rate for visual angles of $100^{\circ}$, $120^{\circ}, 140^{\circ}$, and $160^{\circ}$ ) from the centre of the immersive screen. There were no performance differences in accuracy rate for stimuli presented near to the screen centre between the left $(M$ $=83.3 \%, S D=11.8 \%)$ and right $(M=81.8 \%, S D=10.1 \%)$

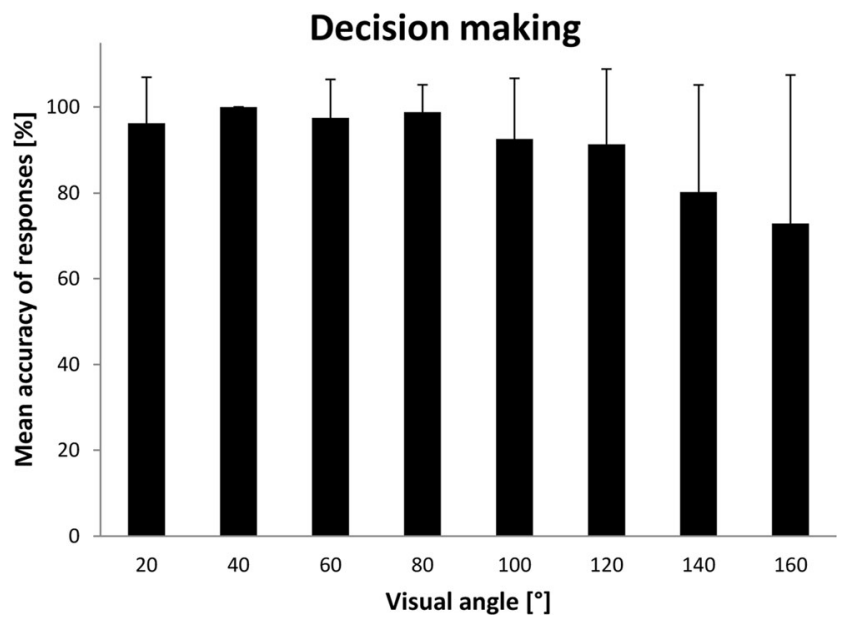

Fig. 4 Percentage of participants' accuracy rate in degrees of visual angle in the motor response decision-making subtask. Symbols represent across-participant means and error bars represent standard deviations
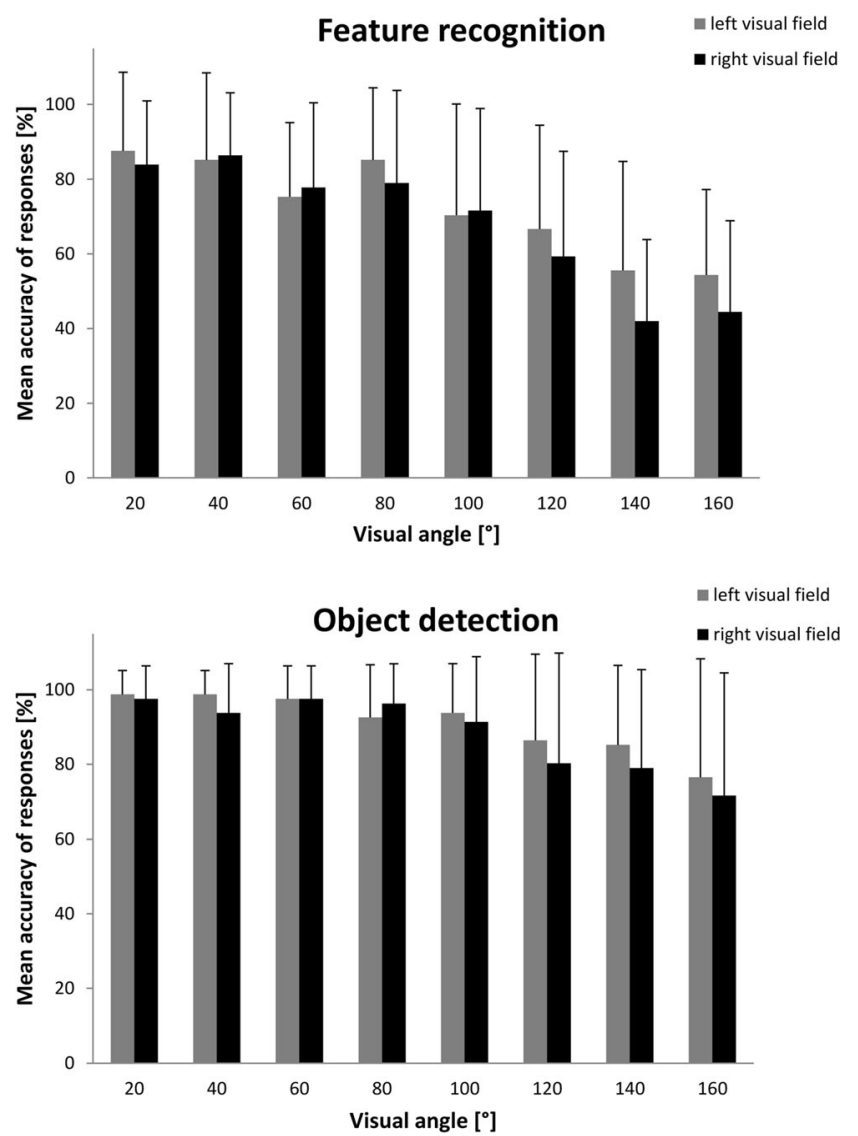

Fig. 5 Percentage of participants' identification rate of the team-mates' running direction (feature-recognition task) and of the number of opponents (object-detection task) in degrees of visual angle as a function of visual field side (left, right). Symbols represent acrossparticipant means and error bars represent standard deviations

visual field side, $t(26)=0.6, p=.574$. However, we found a difference for stimuli presented far away from the screen centre between the left $(M=61.7 \%, S D=18.2 \%)$ and right $(M=$ $54.3 \%, S D=14.3 \%)$ side of the visual field, $t(26)=2.4, p=$ $.024, d=.46$ (Bonferroni-corrected post hoc comparisons had an adjusted alpha of 0.025 ).

Confidence values did not differ between stimuli presented at their left side of visual field $(M=5.6, S D=0.8)$ and those presented at the right side $(M=5.6, S D=0.8)$, $t(26)=1.950, p=.062$.

Object detection subtask In the object-detection task (i.e. perceiving number of opponent players; perception-based task), there was a main effect for (narrow and wide) visual angle, $F(1,26)=14.8, p=.001, \eta^{2}=.36$. Figure 5 (bottom graph) shows that participants' accuracy decreased from the centre to the periphery. Moreover, the ANOVA revealed a main effect of visual field side, $F(1,26)=5.4, p=.028, \eta^{2}=.17$. Participants were more accurate again when perceiving the opponent players at their left side $(M=91.2 \%, S D=9.9 \%)$ compared to those at their right side $(M=88.4 \%, S D=$ 
$11.2 \%)$. We found a significant interaction between visual angle and visual field side, $F(1,26)=7.4, p=.011, \eta^{2}=.22$.

Like in the feature-recognition task, we performed additional t-tests to check if the asymmetrical performance differences in perception existed for stimuli presented both near and far away from the centre of the immersive screen. There were no performance differences in accuracy rate for stimuli presented close to the screen centre between the left $(M=96.9 \%$, $S D=4.7 \%)$ and right $(M=96.3 \%, S D=4.8 \%)$ visual field side, $t(26)=0.6, p=.574$. However, for stimuli presented far away from screen centre the left $(M=85.5 \%, S D=18.1 \%)$ was more accurate than the right $(M=80.6 \%, S D=20.8 \%)$ side of visual field, $t(26)=3.0, p=.005, d=.59$ (Bonferronicorrected post hoc comparisons had again an adjusted alpha of $0.025)$.

In total, participants reported similar confidence levels (left side: $M=6.8, S D=1.1$ right side: $M=6.8, S D=1.1$ ) for their responses on stimuli at both visual field sides, $t(26)=0.3, p=$ .746 , in the object-detection task.

\section{Discussion}

The current study investigated asymmetrical differences in visuospatial perception, attention, and decision making. In accordance with previous research, in near space, participants showed a slight leftwards bias in the basic line-bisection task, known as pseudoneglect (for a review, see Jewell \& McCourt, 2000). In the football task, the same participants achieved higher accuracy rates in the feature-recognition task (perceiving team-mates' running direction) as well as in the objectdetection task (counting number of opponent players) at the left side of the visual field when compared to their right side. This finding, however, contradicts the far-space literature of pseudoneglect (rightward attentional bias) described by Longo and Lourenco (2006, 2007) - at least, at first glance. The authors pointed out that the disparity in spatial visual attention is different for near and far space. Most research has found an attentional bias towards the left side for near space and a change to the right side as one moves to far space. However, in these studies participants mostly used laser pointers to make their judgements (e.g. Longo \& Lourenco, 2006, 2007). When rigid sticks were used for the midpoint estimation, researchers did not observe a left to right shift with increasing distance (e.g. Gamberini, Seraglia, \& Priftis, 2008; Longo \& Lourenco, 2006). In these cases, participants tend to perceive the midpoint of a line as being left of the real midpoint in far space (as they do in near space). This effect was interpreted as an expansion of near space due to tool use (e.g. Lourenco \& Longo, 2009).

While, at first glance, the current results contradict findings on line bisection or target directioning pseudoneglect in far space (at least when considering the wide visual angles in our task), our results cannot be compared directly to previous studies, considering the complex and detailed task used in this study. As opposed to most other research studies concerned with the phenomenon of pseudoneglect, the current study was not about finding the midpoint of the projection (neither by using a laser pointer nor by performing a shot exactly aimed the middle). Rather, this study was about identifying stimuli in the periphery of the left and right visual field at the same time. Thus, the intention of the current study differed markedly from previous studies on asymmetrical attention distribution. Churches and Nicholls (2016) give an overview of findings from different studies conducted in the area of sports so far, as mentioned earlier. Some require a motor response towards an object, whereas others require a motor response towards the middle of two objects. In the study by Roberts and Turnbull (2010), for example, participants aimed at putting a golf ball towards a cup, yet this did not actually require them to bisect a segment of space. As participants in our study were not asked to bisect a segment of space in the football task either, this could explain why our results are more in line with those found by Roberts and Turnbull (2010; rightward deviation) than with other studies involving sport-specific tasks, in which participants were asked to bisect a segment of space, for example, by kicking a ball exactly in the middle between two goal posts (cf. Nicholls et al., 2010). A possible approach for future research, in order to understand attentional and perceptual asymmetries in far space in more detail, might be to ask participants to bisect a segment of space (action task) and to give them tasks requiring perceptual and attentional capabilities at the left and right side of their visual field within the same study.

Furthermore, it might make sense to consider the current results with regard to findings on landmark judgements, which are often used to analyse asymmetrical attention distribution (e.g. Benwell, Thut, Learmonth, \& Harvey, 2013). While the majority of studies dealing with manual bisection have shown a clear slight-left to larger-right bias when stimuli are moved from near to far space (e.g. Longo \& Lourenco, 2006, 2009; Varnava et al., 2002), landmark judgements often point out a transition from a larger-left bias to a smaller-left bias (Bjoertomt, Cowey, \& Walsh, 2002; McCourt \& Garlinghouse, 2000). In addition, studies to date commonly defined far space as the distance forwards into the room/space. In the current study, an immersive screen was used for the first time, so that attentional and perceptual performance, not only in the frontal far space but also in the area lateral to the participants could be analysed. While this would not have made sense in studies on penalty shootouts (cf. Nicholls et al., 2010), this research approach is especially relevant to studies concerning sports games, in which team-mates and opponents have to be perceived across wide visual angles.

One further explanatory approach, which still remains untouched by the heterogeneous findings concerning the 
pseudoneglect effect, and which is already considered by many researchers as a possible cause for the occurrence of leftward perceptual asymmetries (amongst others in facialrecognition and attention), is the direction of reading and writing, characteristic of specific cultures (Megreya \& Havard, 2011; Nicholls \& Roberts, 2002). In this context, it might be interesting to note that spatial representations of relative quantity to different spatial positions are already associated with an oriented space at the start of postnatal life, prior to experience with any culture-specific biases (de Hevia, Veggiotti, Streri, \& Bonn, 2017). A possible explanation of our results might be that participants first processed stimuli on the left side and only later on those stimuli on the right side of their visual field; consequently, in part, not enough time was left for the later stimuli, due to the short presentation periods of $300 \mathrm{~ms}$. This approach would speak for divided attention. Future studies should first of all examine what the attentional distribution in the chosen paradigm looks like exactly, i.e. whether participants form an attentional surface area (cf. zoom-lens model) or divide their attention amongst the relevant objects (cf. divided attention; Hüttermann \& Memmert, 2017).

An interesting finding of the current study is that no differences were found for stimuli in the left and right visual field concerning attentional and perceptual performance for visual angles of up to $80^{\circ}$ in the football-specific decision-making task, which in turn is in line with present research findings (e.g. Gamberini et al., 2008; Longo \& Lourenco, 2006). It is hard to interpret these results on the basis of the present knowledge; they thus only allow for speculation that should be examined in future research. Should the previously described approach, that is participants perceived peripheral stimuli first on the left and second on the right (divided attention, first left, then right), be confirmed, this could be an explanation of why no asymmetries could be found for smaller visual angles. A series of studies already showed that only within an area clearly smaller than the visual field, several stimuli can be consciously perceived at the same time (e.g. Hüttermann \& Memmert, 2017). It may be possible that participants perceived stimuli outside of this attentional area by means of saccades and, consequently, based on their habitual reading direction (form left to right) tried to perceive first the left and then the right stimulus. Due to the limited presentation time of only $300 \mathrm{~ms}$, saccades probably were not possible for all stimuli outside the attentional area. Here too, analyses of gaze behaviour by means of eye-tracking systems are desirable in future research.

There might be further alternative interpretations of the current findings that should be considered by future research in more detail as well. One approach might be the two visualsystems model by Milner and Goodale (1995, 2008). According to this approach, any misbisection could be justified by the existence of two functionally and neuroanatomically dissociated systems of stimulus information processing for perception and action, with attentional asymmetries only occurring for the perception system (cf. Hughes, Bates, \& Aimola Davies, 2004, 2008). Although, at first sight, the current task could be assigned to the perception system (verbally performed object-detection and featurerecognition task) than to the relatively easier decisionmaking task (pass to the left, right, or no pass), which would be dependent on the action system, previous research has demonstrated that far-aiming tasks (e.g. kicking/throwing objects to targets at distance) rely on the perception system as well (e.g. Shim et al., 2014; van der Kamp, van Dorn, \& Masters, 2009). Future research and analyses are needed to investigate the link between attentional/perceptual capabilities at a person's right and left side of the visual field and the two visual-systems model in more detail.

Previous studies that have used the same method (cf. Hüttermann, Ford, et al., 2019a; Hüttermann, Smeeton, et al., 2019b) have not found a positive correlation between performance on the football task and a working memory task (Aospan task; cf. Unsworth, Heitz, Schrock, \& Engle, 2005). The results from the football task can therefore be attributed to attentional and perceptual capabilities, rather than to working memory capacity.

In addition to the asymmetries in spatial attention and perception, the current study also investigated athletes' decision making in the football-specific task. Similar to the results of the studies of Hüttermann and colleagues (cf. Hüttermann, Ford, et al., 2019a; Hüttermann, Smeeton, et al., 2019b), participants chose the right solution in $91 \%$ of all game situations (pass to one side or controlling the ball), even when they gave the wrong answer in the feature-recognition and objectdetection task. This can be explained by the fact that, according to the participants themselves, they could often detect whether team-mates in the periphery were surrounded by opponent players or not, even when they could not verbalise the running direction of the team-mates and the number of opponent players correctly. However, it should be considered that attentional processes in real-life game situations can be much more complex than the challenge to decide whether to pass the ball to the left, to the right, or whether to control it/not pass at all. Here, for example, decisions have to be made about how to play the ball (distinction between different techniques, e.g. low pass, return pass, dribble), with which speed and force to play the ball (amongst others the running speed of a teammate has to be considered), and possibly even more teammates and opponents have to be included into the decisionmaking process. In these cases, attentional and perceptual skills become even more important for the decision-making process. Future studies should take this into account. Additionally, in order to examine a larger number of visual angles, the number of trials per angle was reduced. The main effect of visual angle across all tasks demonstrates reduced performance at larger eccentricities and is consistent with 
other studies (e.g. Hüttermann, Ford, et al., 2019a; Hüttermann, Smeeton, et al., 2019b). However, future studies could reduce the number of visual angles and examine a larger number of trials per angle to more precisely measure the visual angle threshold between success and failure at the tasks.

For several years, there is the commitment of several scientists in sport (psychology) research for more ecological validity and representative designs of experimental paradigms (cf. Dicks, Davids, \& Button, 2009). This is a necessary step to maximize the transferability of findings from laboratory settings to performance environments, such as in sports (cf. Brunswik, 1956). Especially in sport science where the importance of the ecological approach is constantly growing (cf. Araújo, Davids, \& Passos, 2007), athletes' performances should be analysed in representative designs of experimental paradigms (e.g. Hüttermann, Memmert, \& Liesner, 2014). A large immersive concave screen - as was used in the current study - preserves the need for a broad attentional focus that is required for many decision-making tasks in sport (cf. Hüttermann \& Memmert, 2017; Klatt \& Smeeton, 2019).

Even though this study required decision behaviour from the participants regarding the passing direction or controlling of the ball, no statements can be made on the extent to which the asymmetrical orientation of perceptual and attentional capabilities had an impact on the decision behaviour. This is connected to the fact that in each trial only one decision had to be made. Subsequent works of research should, thus, modify the task in a way that decisional performance would be required for the left and the right visual field independently. Furthermore, future studies should recruit a sample ensuring a balance between left- and right-footed shooters. Due to the fact that only four left-footed players and 23 right-footed players participated in the current study, meaningful analyses were impossible. Future research should pursue the approach that preferences of the preferred shooting foot might affect the visual field side (left, right) in motor tasks - relying on insights that motor areas of the brain control opposite sides (i.e. primary motor cortex on the left side of the brain controls movements of the right side of the body, and vice versa, the right motor cortex controls movements of the left side of the body; Serrien, Ivry, \& Swinnen, 2006).

In summary, the heterogeneity and low number of studies investigating the pseudoneglect effect, especially for tasks that go beyond a simple line-bisection task, indicate that the observed differences in results need to be interpreted with caution. Inter- and intra-individual differences may explain the variety of outcomes seen. The findings of the current study indicate that the movements and positioning of team-mates and opponents on the player's right side of the visual field are perceived inferiorly to those on the player's left side, at least for large visual angles. While this can pose an advantage for the opponents, who will thereby more frequently evade the player's focus, team-mates should try to position themselves in a way that they will be perceived by the player and can be included into the decision-making process; this is more likely on the left than on the right side of the player's visual field. Future research is still needed to analyse to what extent visual asymmetries do influence players' decision making dependent on the complexity of game situations.

\section{References}

Araújo, D., Davids, K., \& Passos, P. (2007). Ecological validity, representative design, and correspondence between experimental task constraints and behavioral setting: comment on Rogers, Kadar, and Costall (2005). Ecological Psychology, 19, 69-78.

Benedetto, S., Pedrotti, M., Bremond, R., \& Baccino, T. (2013). Leftward attentional bias in a simulated driving task. Transportation Research Part F: Traffic Psychology and Behaviour, 20, 147-153.

Benwell, C. S., Thut, G., Learmonth, G., \& Harvey, M. (2013). Spatial attention: differential shifts in pseudoneglect direction with time-ontask and initial bias support the idea of observer subtypes, Neuropsychologia, 51, 2747-2756.

Bjoertomt, O., Cowey, A., \& Walsh, V. (2002). Spatial neglect in near and far space investigated by repetitive transcranial magnetic stimulation. Brain, 125, 2012-2022.

Bowers, D., \& Heilman, K. M. (1980). Pseudoneglect: effects of hemispace on a tactile line-bisection task. Neuropsychologia, 18, 491-498.

Brunswik, E. (1956). Perception and the representative design of psychological experiments (2nd ed.). Berkeley: University of California Press.

Bultitude, J. \& Aimola Davies, A. M. (2006). Putting attention on the line: Investigating the activation-orientation hypothesis of pseudoneglect. Neuropsychologia, 44, 1849-1858.

Cai, Q., van der Haegen, L., \& Brysbaert, M. (2013). Complementary hemispheric specialization for language production and visuospatial attention. Proceedings of the National Academy of Sciences, 110, 322-330.

Churches, O., \& Nicholls, M. E. R. (2016). Near Misses and the Effect of Attentional Asymmetries on Sporting Performance. In: F. Loffing, N. Hagemann, B. Strauss, \& C. MacMahon (Eds.), Laterality in sports (pp. 205-224). San Diego: Academic Press.

Corballis, M. C. (1989). Laterality and human evolution. Psychological Review, 96, 492-505.

Corbetta, M., \& Shulman, G. L. (2011). Spatial neglect and attention networks. Annual Review of Neuroscience, 34, 569-599.

De Hevia, M. D., Veggiotti, L., Streri, A., \& Bonn, C. (2017). At birth humans associate "few" with left and "many" with right. Current Biology, 27, 3879-3884.

Dehaene, S., Changeux, J. P., Naccache, L., Sackur, J., \& Sergent, C. (2006). Conscious, preconscious, and subliminal processing: A testable taxonomy. Trends in Cognitive Sciences, 10, 204-211.

Dellatolas, G., Vanluchene, J., \& Coutin, T. (1996). Visual and motor components in simple line bisection: An investigation in normal adults. Cognitive Brain Research, 4, 49-56.

Dicks, M., Davids, K., \& Button, C. (2009). Representative task designs for the study of perception and action in sport. International Journal of Sport Psychology 40, 506-524.

Driver, J., \& Mattingley, J. B. (1998). Parietal neglect and visual awareness. Nature Neuroscience, 1, 17-22.

Fink, G. R., Marshall, J. C., Shah, N. J., Weiss, P. H., Halligan, P. W., Grosse-Ruyken, M., et al (2000). Line bisection judgments 
implicate right parietal cortex and cerebellum as assessed by fMRI. Neurology, 28, 1324-1331.

Fink, G. R., Marshall, J. C., Weiss, P. H., Toni, I., \& Zilles, K. (2002). Task instructions influence the cognitive strategies involved in line bisection judgements: evidence from modulated neural mechanisms revealed by fMRI. Neuropsychologia, 40, 119-130.

Frasnelli, E., Vallortigara, G., \& Rogers, L. J. (2012). Left-right asymmetries of behaviour and nervous system in invertebrates. Neuroscience \& Biobehavioral Reviews. 36, 1273-1291.

Gamberini, L., Seraglia, B., \& Priftis, K. (2008). Processing of peripersonal and extrapersonal space using tools: Evidence from visual line bisection in real and virtual environments. Neuropsychologia, 46, 1298-1304.

Holtzman, J. D., Sidtis, J. J., Volpe, B. T., Wilson, D. H., \& Gazzaniga, M. S. (1981). Dissociation of spatial information for stimulus localization and the control of attention. Brain, 104, 861-872.

Hughes, L. E., Bates, T. C., \& Aimola Davies, A. M. (2008). Dissociations in rod bisection: The effect of viewing conditions on perception and action. Cortex, 44, 1279-1287.

Hughes, L. E., Bates, T. E., \& Aimola Davies, A. M. (2004). Grasping at sticks: Dissociations between perception and action. Experimental Brain Research, 157, 397-402.

Hüttermann, S., \& Memmert, D. (2017). The attention window: A narrative review of limitations and opportunities influencing the focus of attention. Research Quarterly for Exercise and Sport, 88, 169-183.

Hüttermann, S., Ford, P. R., Williams, A. M., Varga, M., \& Smeeton, N. J. (2019). Vision, perception, and action in a simulated decisionmaking task. Journal of Sport \& Exercise Psychology, 41, 230-241.

Hüttermann, S., Helsen, W. F., Put, K., \& Memmert, D. (2018). Does visual attention impact on decision-making in complex dynamic events? Journal of Sport \& Exercise Psychology, 40, 163-166.

Hüttermann, S., Memmert, D., \& Nerb, J. (2019). Individual differences in attentional capability are linked to creative decision making. Journal of Applied Social Psychology, 49, 159-167.

Hüttermann, S., Memmert, D., \& Simons, D. J. (2014). The size and shape of the attentional "spotlight" varies with differences in sports expertise. Journal of Experimental Psychology: Applied, 20, 147157.

Hüttermann, S., Memmert, D., \& Liesner, F. (2014). Finding the happy medium: An analysis of gaze behavior strategies in a representative task design of soccer penalties. Journal of Applied Sport Psychology, 26, 172-181.

Hüttermann, S., Memmert, D., Simons, D. J., \& Bock, O. (2013). Fixation strategy influences the ability to focus attention on two spatially separate objects. PLoS ONE, 8, e65673.

Hüttermann, S., Noël, B., \& Memmert, D. (2017). Evaluating erroneous offside calls in soccer. PLOS ONE, 12, e0174358.

Hüttermann, S., Smeeton, N. J., Ford, P. R., \& Williams, A. M. (2019). Color perception and attentional load in dynamic, time-constrained environments. Frontiers in Psychology, 9, 2614.

Jewell, G., \& McCourt, M. E. (2000). Pseudoneglect: A review and metaanalysis of performance factors in line-bisection tasks. Neuropsychologia, 38, 93-110.

Klatt, S., \& Smeeton, N. J. (2019). Immersive screens change attention width but not perception or decision-making performance in natural and basic tasks. Applied Ergonomics, 82, 102961.

Lamme, V. A. (2003). Why visual attention and awareness are different. Trends in Cognitive Sciences, 7, 12-18.

Lippolis, G., Bisazza, A., Rogers, L. J., \& Vallortigara, G. (2002). Lateralization of predator avoidance responses in three species of toads. Laterality, 7, 163-183.

Longo, M. R., \& Lourenco, F. S. (2006). On the nature of near and far space: Effects of tool use and the transition to far space. Neuropsychologia, 44, 977-981.
Longo, M. R., \& Lourenco, S. F. (2007). Space perception and body morphology: Extent of near space scales with arm length. Experimental Brain Research, 177, 285-290.

Lourenco, F. S., \& Longo, M. R. (2009). The plasticity of near space: Evidence for contraction. Cognition, 112, 451-456.

MacNeilage, P. F., Rogers, L. J., \& Vallortigara, G. (2009). Origins of the left and right Brain. Scientific American, 301, 60-67.

Masters, R. S. W., van der Kamp, J., \& Jackson, R. C. (2007). Imperceptibly off-center goalkeepers influence penalty-kick direction in soccer. Psychological Science, 18, 222-223.

McCourt, M. E., \& Jewell, G. (1999) Visuospatial attention in line bisection: stimulus modulation of pseudoneglect. Neuropsychologia, 37, 843-855.

McCourt, M., \& Garlinghouse, M. A. (2000). Stimulus modulation of pseudoneglect: Influence of line geometry. Neuropsychologia, 38, 520-524.

Megreya, A. M., \& Havard, C. (2011). Left face matching bias: Right hemisphere dominance or scanning habits? Laterality: Asymmetries of Body, Brain and Cognition, 16, 75-92.

Milner, A. D., \& Goodale, M. A. (1995). The visual brain in action. Oxford: Oxford University Press.

Milner, A. D., \& Goodale, M. A. (2008). Two visual systems re-viewed. Neuropsychologia, 46, 774-785.

Milner, B. (1975). Psychological aspects of focal epilepsy and its neurosurgical management. In D. P. Purpura, J. K. Penry \& R. D. Walter (Eds.), Advances in neurology, Vol. 8 (pp. 299-321). New York: Raven.

Nicholls, M. E., Loftus, A. M., Orr, C. A., \& Barre, N. (2008). Rightward collisions and their association with pseudoneglect. Brain and Cognition, 68, 166-170.

Nicholls, M. E., \& Roberts, G. R. (2002). Can free-viewing perceptual asymmetries be explained by scanning, pre-motor or attentional biases? Cortex, 38, 113-136.

Nicholls, M. E., Loetscher, T., \& Rademacher, M. (2010). Miss to the right: The effect of attentional asymmetries on goal-kicking. PLoS One, 5, e12363.

Noël, B., van der Kamp, J., Weigelt, M., \& Memmert, D. (2015). Asymmetries in spatial perception are more prevalent under explicit than implicit attention. Consciousness and Cognition, 34, 10-15.

Nottebohm, F., Stokes, T. M., \& Leonard, C. M. (1976). Central control of song in the canary, Serinus canarius. The Journal of Comparative Neurology, 165, 457-486.

Roberts, R., \& Turnbull, O. H. (2010). Putts that get missed on the right: Investigating lateralized attentional biases and the nature of putting errors in golf. Journal of Sports Sciences, 28, 369-374.

Rogers, L. J., Vallortigara, G., \& Andrew, R. J. (2013). Divided brains: The biology and behaviour of brain asymmetries. New York, NY, US: Cambridge University Press.

Rogers, L. J., Zucca, P., \& Vallortigara, G. (2004) Advantage of having a lateralized brain. Proceedings of the Royal Society of London, B 271, S420-S422.

Serrien, D. J., Ivry, R. B., \& Swinnen, S. P. (2006). Dynamics of hemispheric specialization and integration in the context of motor control. Nature Reviews Neuroscience, 7, 160-166.

Shim, J., van der Kamp, J., Rigby, B. R., Lutz, R., Poolton, J. M., \& Masters, R. S. W. (2014). Taking aim at the Müller-Lyer goalkeeper illusion: An illusion bias in action that originates from the target not being optically specified. Journal of Experimental Psychology: Human Perception and Performance, 40, 1274-1281.

Unsworth, N., Heitz, R.P., Schrock, J.C., \& Engle, R.W. (2005). An automated version of the operation span task. Behavior Research Method, 37, 498-505.

Vallortiga, G., \& Rogers, L. J. (2005). Survival with an asymmetrical brain: Advantages and disadvantages of cerebral lateralization. Behavioural and Brain Sciences, 28, 575-633. 
Vallortigara, G., Rogers, L. J., Bisazza, A., Lippolis, G., \& Robins, A. (1998). Complementary right and left hemifield use for predatory and agonistic behaviour in toads. Neuroreport, 9, 3341-3344.

van Boxtel, J. J. A., Tsuchiya, N., \& Koch, C. (2010). Consciousness and attention: On sufficiency and necessity. Frontiers in Psychology, 1, 217.

van der Kamp, J., van Doorn, H., \& Masters, R. S. W. (2009). A Judd illusion in far-aiming: Evidence of a contribution to action by vision for perception. Experimental Brain Research, 197, 199-204.

Varnava, A., \& Halligan, P. W. (2008). Comparative measures of linear extent: implications for clinical assessment. Applied Neuropsychology, 15, 107-112.

Varnava, A., McCarthy, M., \& Beaumont, J. G. (2002). Line bisection in normal adults: Direction of attentional bias for near and far space. Neuropsychologia, 40, 1372-1378.
Verleger, R., \& Śmigasiewicz, K. (2015). Consciousness wanted, attention found: Reasons for the advantage of the left visual field in identifying T2 among rapidly presented series. Consciousness and Cognition, 35, 260-273.

Weigelt, M., \& Memmert, D. (2012). Goal-side selection in soccer penalty kicking when viewing natural scenes. Frontiers in Psychology, 3, 312 .

Weiss, P. H., Marshall, J. C., Wunderlich, G., Tellmann, L., Halligan, P. W., Freund, H. J., et al (2000). Neural consequences of acting in near versus far space: A physiological basis for clinical dissociations. Brain, 123, 2531-2541.

Publisher's note Springer Nature remains neutral with regard to jurisdictional claims in published maps and institutional affiliations. 